\title{
Improving Care of Critically Unwell Patients through Development of a Simulation Programme in a Malawian Hospital
}

\author{
Jonathan Barnes ${ }^{1,2}$, Lucy Paterson-Brown ${ }^{1,3}$ \\ ${ }^{1}$ Fistula Care Centre, Bwaila Maternity Hospital, Lilongwe, Malawi \\ ${ }^{2}$ Department of Intensive Care Medicine, University College Hospital, 235 Euston Road, London, UK \\ ${ }^{3}$ Department of Paediatrics, Croydon University Hospital, 530 London Road, London, UK \\ Correspondence: Jonathan Barnes, Department of Intensive Care Medicine, University College Hospital, 235 Euston \\ Road, London, UK.
}

Received: March 9, 2017

Accepted: April 20, 2017 Online Published: May 3, 2017

doi:10.11114/jets.v5i6.2366

URL: https://doi.org/10.11114/jets.v5i6.2366

\begin{abstract}
Introduction: Malawi is one of the world's poorest countries with very limited healthcare spending and a lack of post-graduate training for healthcare workers, including in critical illness management. Critical illness simulation courses have been shown to be an effective training tool and form a key part of training for healthcare professionals in the developed world. However, these courses can be difficult and costly to set. We therefore developed an affordable and simple to run programme for use in a regional hospital in Malawi.

Methods: We delivered a series of lectures alongside a simulation course based around critical illness management, including cardio-pulmonary-resuscitation. The course was taught by a visiting physician who trained a local clinical officer to continue running the course in the future. Feedback on candidate confidence in managing acutely unwell patients was collected before and after the course.

Results: All staff in the unit attended the course. Median candidate confidence across all seven assessed areas increased significantly.

Conclusions: Despite the basic equipment used in our course, we received very positive feedback and feel that this highlights that significant improvements in staff skillset, and therefore patient outcomes, can be achieved using a relatively simple and low cost intervention that can also easily be continued by local members of staff, ensuring sustainability. Introduction of similar courses in other resource poor settings may help improve patient outcomes without the significant financial outlay and lack of future-proofing seen with many healthcare initiatives that are deployed, and are often unsuccessful, in the developing world.
\end{abstract}

Keywords: simulation, acute, critical, Malawi, Africa, fistula

\section{Introduction}

\subsection{Background to Malawian Healthcare}

Malawi is one of the world's poorest countries with healthcare spending of under $\$ 100$ per capita, per year (World Health Organisation, WHO, 2014). This is in comparison to approximately $\$ 3500$ spent in the UK (WHO, 2015). In association with very limited spending, Malawi has only 0.02 physicians per 1000 population, again this is in stark contrast to the UK where the number is approximately 150 times greater (WHO 2014, WHO 2015).

This resource limitation impacts not only on how readily the population can access healthcare services, but also on healthcare workers' ability to access on going post-graduate education and training, which is recognised as crucial for the development of high quality healthcare services (WHO, 2001, Davis, 1998). This problem is highlighted by the fact that hospital staff across the country do not routinely have access to any acute illness management or resuscitation training, which would be considered obligatory for clinical staff in more developed countries. Indeed, a lack of adequate continued professional development and post-graduate training has been highlighted as a problem in the Malawian health system in previous work (Muula et al., 2004) with one study highlighting that healthcare workers, including nurses, "consider current continuous education and career progression strategies [in Malawi] to be inadequate"(Manafa et al., 2009). 


\subsection{Background to Simulation Training}

Acute illness management courses, which are predominantly simulation based, enable healthcare workers to become familiar with recognising and managing various critical illness scenarios and performing various life saving procedures, including cardio-pulmonary-resuscitation (CPR). They form a key part of training for all medical, nursing and allied healthcare professionals in the developed world and evidence suggests that such training improves confidence in managing emergency situations (Hogg \& Miller, 2016), clinical acumen and skillset (Ruessler et al., 2010, Theilen et al., 2013, Cant \& Cooper, 2010) alongside actual patient care (Theilen et al., 2013), safety and outcomes (Zendejas, Brydges, Wang, Cook, 2013). Simulation has specifically been shown to be an innovative and useful tool in nursing education (Chen, Huang, Liao, Liu, 2015), where it can lead to "gains in knowledge, critical thinking, satisfaction and confidence" (Cant \& Cooper, 2010). Such training may be particularly valuable in low resource settings, such as Malawi, where training opportunities are limited.

Key limitations to implementation of such course include lack of staff qualified to act as instructors and a lack of resources (Huang, Gordan, Schwartzstein, 2007). The often prohibitively expensive and immobile nature of the equipment (e.g. simulation mannequins in simulation centres) required to teach simulation courses is a particular issue in low resource settings.

\subsection{Introduction to Our Centre and Patients}

The Fistula Care Centre at Bwaila Hospital in Lilongwe, Malawi, currently performs over 400 operations per year. Predominantly these are obstetric fistula repairs, but the caseload also includes more complex colorectal and urological procedures (e.g. urinary diversions). Due to the medical and social sequalae of obstetric fistula, patients often present for surgery malnourished and with some degree of renal impairment, both of which are recognised predictors of poor surgical outcome (Culebras, 2013, Mooney, Chow, Hillis, 2014). Furthermore, the low wages paid to most in the country and the consequent unaffordability of even basic medications mean that many patients are also suffering from other untreated chronic illness (e.g. diabetes mellitus, hypertension), which further adds to the burden of peri- and post-operative morbidity and mortality (Abdullah \& Al-Salamah, 2009). Furthermore, many interventions that are seen as routine practice to minimise post-operative complications in the developed world (e.g. venous-thromboembolism chemical prophylaxis, specialist pain team input, nosocomial infection screening etc.) simply do not exist in Malawi and many other similar resource poor healthcare systems. This further adds to the risk of adverse surgical outcomes and post-operative complications. It is therefore vital that the nursing staff at the Fistula Care Centre have an ability to recognise and intervene in the acutely unwell and deteriorating patient. However, lack of resource availability, means that training in this vital area is grossly underprovided.

\subsection{This Project}

We aimed to develop a training programme that would equip the nursing staff at the Fistula Care Centre with the clinical knowledge, practical skillset and communication skills to recognise and manage the acutely unwell patient and know when and how to call for help. Given the low resource setting, we needed to ensure that the course was financially viable and logistically implementable and that it was sustainable and could continue to be run in the future.

We developed a course that would equip nurses with the core skills required for emergency nursing, based on evidence identified in previous literature (Carroll, 2004), and the essential practical skills and communication components of basic life support (Resuscitation Council, 2016).

\section{Methods}

\subsection{The Course}

We developed a programme comprising a series of lectures covering critical-illness based topics, supplemented by a half day simulation course in which candidates were asked to recognise and manage basic medical emergencies, including performing CPR. The course used the principle of ABCDE (airway, breathing, circulation, disability, exposure) for assessment and SBAR (situation, background, assessment, response) for handover, as advocated by the UK resuscitation council (Resuscitation Council, 2016).

The lectures covered recognition of the sick patient (including ABCDE and SBAR), sepsis and shock, diabetic emergencies, post operative respiratory complications (including opioid toxicity and anaphylaxis), principles of fluid management, renal failure, seizures and haemorrhage management. The curriculum was reviewed, to ensure appropriateness and relevance, by the centre's senior medical staff and clinical lead.

During the simulation sessions the principles of $\mathrm{ABCDE}$ assessment and SBAR handover were reviewed and practiced. Basic airway management skills and CPR were also taught and practiced using a mannequin (Resusci-Annie, Laerdal, Stavanger, Norway), which was sourced from a local clinic free of charge. Each nurse was then given two emergency 
medical scenarios to manage. They were given a clinical vignette and then asked to carry out an ABCDE assessment on the mannequin (observations/responses were prompted by the facilitator), implement a basic management plan and then handover using an SBAR technique. The patient would then deteriorate requiring re-assessment and CPR.

The course was taught by a visiting physician with the assistance of a local clinical officer. Lectures were delivered to the whole nursing cohort whilst the simulation course taught to small groups of three to four at a time.

The clinical officer observed the initial sessions before delivering teaching sessions under observation and then independently with the goal that this individual would then be equipped to train more nurses in the future and continue to run the course on an annual basis.
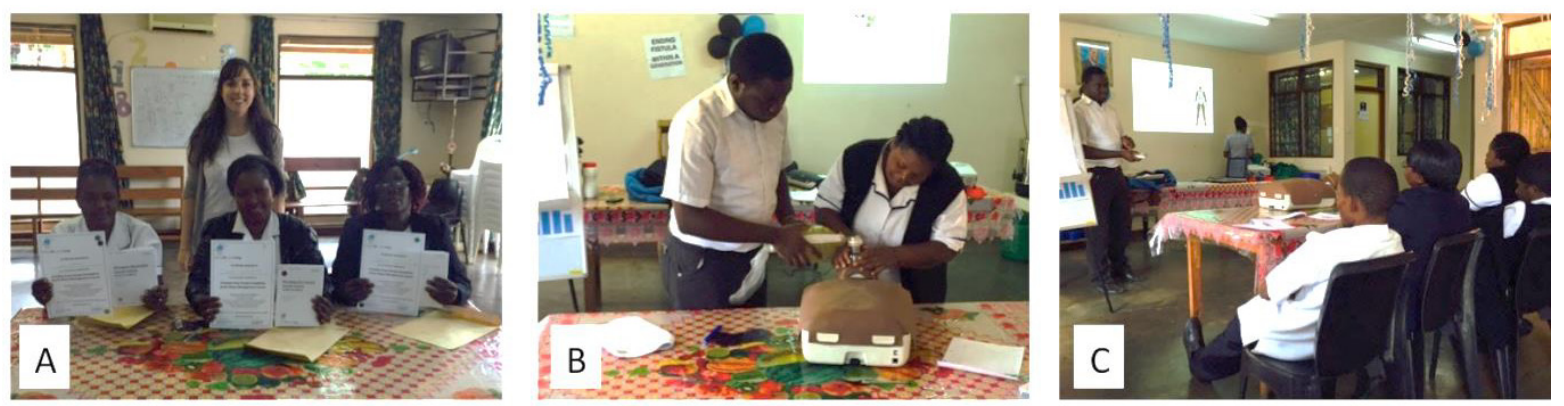

Figure 1. Visiting physician and candidates with their course certificates (A), local clinical officer teaching a candidate bag-valve-mask ventilation (B), candidates attending a lecture delivered by the clinical officer (C).

Participants were provided with a course handbook and a series of accompanying "emergency management protocols" were developed for use on the wards. The same content was delivered at each session.

The course was piloted in early 2015 and received extremely positive feedback from the participants and the senior clinical and management team at the centre. After this success, it was repeated the following year with formal candidate feedback sought to validate the findings of the 2015 programme.

\subsection{Course Evaluation}

Candidates were given a pre and post course questionnaire to evaluate whether the course had led to a change in confidence in relation to recognising and managing acutely unwell patients.

The evaluation tool used was a novel tool developed by the authors specifically for use with our study population. The areas evaluated for subject confidence were selected based on a recent study that identified several essential skills for nurses working in the emergency setting (Munroe et al., 2016), specifically; history taking, assessment and identification of red flags, interventions, diagnostics, reassessment and communication. These areas were also identified in a meta-analysis as some of the core skills for nurses working in the acute setting (Carroll, 2004) adding validity to the assessment tool. The assessment tool also incorporated assessment of the essential basic skills taught in Advanced Life Support (Resuscitation Council, 2016).

Our tool evaluated candidates' confidence in;

- Recognising the sick patient (history and assessment)

- Assessing the sick patient (specifically the ABCDE system)

- Emergency management interventions and procedures

- Communication in the emergency setting (specifically the SBAR handover)

- Recovery position

- Basic airway manaueveres and management

- Cardio-pulmonary resuscitation

For purposes of analysis, the self-assessment questionnaire was in English. Although all of the candidates spoke English and used it in the centre, it was not their first language. Therefore, to avoid any confusion or erroneous results, we developed a tool employing simple language and using a visual analogue scale to assess confidence (from one - not confident, to five - very confident). Alongside this, the centre's head nurse assisted by offering further explanation of the questions in the candidates' local language if there was any confusion. 


\subsection{Sample Selection}

All nursing staff working in the centre were invited to attend the course and were provided with a pre and post course evaluation.

\subsection{Statistical Analysis}

Data was collected and entered into and analysed using Microsoft Excel (Microsoft Excel for Mac, 2011, Microsoft, Washington) with Statplus:mac (Analysoft, California).

Data was analysed for statistical significance using a Wilcoxin signed-rank test with Z-calculations.

\section{Results}

\subsection{Programme Sample}

The course was attended by all 20 of the nursing staff at the centre (100\% of invitees). Of the 20 , two were male (10\%), 18 were female (90\%). All were Malawian nationals who had trained in Malawi.

\subsection{Programme Results}

Feedback was collected from all candidates. Candidate confidence improved significantly across all domains after attending the training course, see table 1 and figure 2 .

Table 1. Median self-reported confidence across different areas of acute care, before and after attending the acute illness management course.

\begin{tabular}{|c|c|c|c|c|c|c|c|}
\hline & \multicolumn{2}{|c|}{ Pre Course Score } & \multicolumn{5}{|c|}{ Post Course Score } \\
\hline & Median & Range & Median & Range & $\begin{array}{l}\text { Relative } \\
\text { confidence } \\
\text { change (\%) }\end{array}$ & $Z$ value & P value \\
\hline Recognising the sick patient & 4 & $3-5$ & 5 & $3-5$ & $+25.0 \%$ & -3.18 & 0.0015 \\
\hline (ABCDE) & 3 & $2-5$ & 5 & $3-5$ & $+66.7 \%$ & -3.62 & 0.0003 \\
\hline Management & & & & & & & \\
\hline Procedures & 4 & $2-5$ & 5 & $3-5$ & $+25.0 \%$ & -3.08 & 0.002 \\
\hline SBAR Handover & 3 & $1-5$ & 5 & $3-5$ & $+66.7 \%$ & -3.70 & 0.0002 \\
\hline Recovery Position & 4 & $2-5$ & 5 & $3-5$ & $+25.0 \%$ & -3.15 & 0.0016 \\
\hline Basic Airway Manouveres & 3 & $1-5$ & 5 & $3-5$ & $+66.7 \%$ & -3.34 & 0.0008 \\
\hline CPR & 3 & $1-5$ & 5 & $3-5$ & $+66.7 \%$ & -3.62 & 0.0003 \\
\hline
\end{tabular}

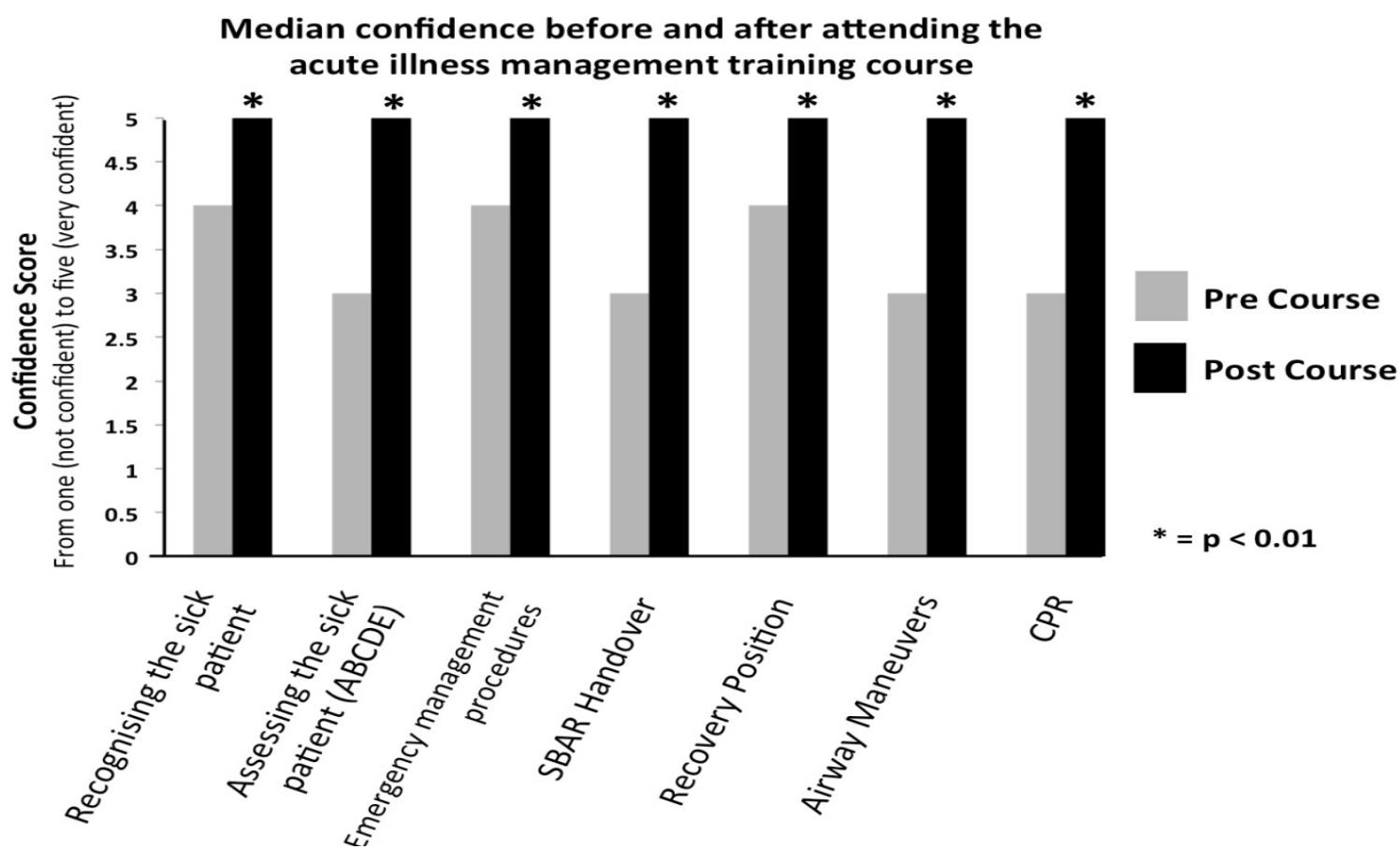

Figure 2. Candidate self reported confidence before and after attending the course.

The median overall candidate confidence in managing acutely unwell patients (assessed across all domains) improved from three before attending the course, to five post course ( $66.7 \%$ relative increase), $\mathrm{p}<0.01$. 


\section{Conclusions}

\subsection{Summary of Findings}

Simulation courses have been shown to be an effective tool in critical illness management training (Hogg \& Miller, 2016, Ruesseler et al., 2010, Theilen et al., 2013, Cant \& Cooper, 2010, Zendejas et al., 2013) and therefore may be of particular value in low resource settings, like Malawi, where access to on-going professional development is known to be limited (Muula et al., 2004, Manafa et al., 2009). However, simulation courses can be difficult to set up due to the nature of the equipment involved and the requirement for instructors, which can be a particular issue in low resource settings.

We used a simple resuscitation doll with faculty input to give observations and findings to develop a simulation based acute care course for use in the Bwaila Fistula Care Centre, a centre that does deal with acutely unwell (normally post-operative) patients, but where the nursing staff do not have access to formal training in this area due to a lack of resources/availability of instructors.

Despite the relatively rudimentary equipment used in our course, we received very positive feedback from senior medical and nursing staff and observed a significant improvement in candidate confidence across various areas of acute care, after attending the course. These results are in keeping with other literature from more developed settings, where high fidelity simulation has been used (Hogg \& Miller, 2016), suggesting comparability of programme outcomes. The findings in this study were consistent with the informal feedback we had received the previous year when we ran a pilot programme, suggesting validity of our programme and probable reproducibility of results. We feel that this demonstrates that even with only basic equipment significant improvements in nursing staff knowledge and understanding relating to acute illness management can be achieved, which we believe will translate into better patient care, clinical outcomes and staff satisfaction.

By training a local member of the clinical staff as an instructor, alongside delivering the course, we plan that the course will become established as a regular feature in the continuing professional development calendar for all nursing staff at the centre. Because the course is an original course, devised by the authors, there are no on-going licensing costs to be incurred, which should allow the course to continue to run without further, potentially prohibitive, costs to the centre, a known barrier to simulation training (Chen et al., 2015).

The areas evaluated by our assessment tool were in keeping with areas previously identified as core skills for nursing staff (Carroll, 2004, Resuscitation Council, 2016, Munroe et al., 2016), adding validity to our assessment instrument, and both the format of the course and the areas of assessment were comparable to more recently published work looking at the effect of simulation training for medical students (Hogg \& Miller, 2016), again adding validity to the methodology and results of this work.

\subsection{Limitations}

Although our results are strongly positive, this study is limited by the fact that all of our data is self-reported and subjective. A different approach to assessment of the course, which would potentially give more valuable results, would be for candidates to undertake some form of assessment (perhaps an observed simulated clinical encounter and a written test) before and after the course. This would allow for generation of objective data regarding the effectiveness of this course. However, this was beyond the scope of this current work although would be a valuable area for future assessment. Despite this, we feel that the fact that this course did lead to a significant improvement in nursing staff clinical confidence, in keeping with results from more elaborate and established programmes (Hogg \& Miller, 2016), suggests that the objective improvements in clinical practice and clinical outcome that simulation can lead to (Ruesseler et al., 2010, Theilen et al., 2013, Cant \& Cooper, 2010, Zendejas et al., 2013) should be realisable from this course.

It is also possible that candidates' responses were biased and that they over-reported their post course confidence as they were working with the instructors on a daily basis as part of their normal clinical work. However, we attempted to mediate this by anonymising the questionnaires and insisting that candidates be honest. A final limitation of this study is its small sample size, even given the extremely strongly positive results. Further work would benefit from recruitment of a larger sample size.

\subsection{Suggestions for Future Work}

Further evaluation of this course would be useful. An actual evaluation of the course itself, using a validated instrument, may be valuable, particularly in identifying areas for development/improvement. Equally, evaluation of the course outcomes using some form of objective assessment of candidate performance and a larger study group, achieved perhaps by introducing the course into other similar centres as a multi-centre programme, would be of value. It would also be useful to assess candidates self reported ability and confidence, and general approach to practise, further down the line (e.g. at one year) to evaluate whether candidates had retained, or even built on, the skills and approaches 
developed during the course. This longtitudinal follow up would also be useful in assessing how regularly the training needs to be repeated in order to ensure skillset retention. A final area that would be extremely valuable to evaluate would be patient outcomes, particularly in regard to post-operative morbidity and mortality, before and after the implementation of this course. Although this would be an area with multiple confounders it may be of great value.

\subsection{Conclusion}

Despite the various limitations of this study, we feel that it highlights that significant improvements in nursing staff skillset, and therefore patient outcomes, can be achieved using a relatively simple and low cost intervention, in a low resource setting. This programme was not only effective, but it can also be easily carried on by members of the local clinical staff, ensuring sustainability.

Introduction of similar courses in other low resource settings may help improve outcomes without the significant financial outlay and lack of future-proofing seen with many healthcare initiatives that are deployed, and are often unsuccessful, in the developing world.

\section{Acknowledgements}

We would like to thank the Freedom from Fistula Foundation and the Gloag Foundation for supporting our work in Malawi and supporting the on going vital work carried out by the rest of the team at the Fistula Care Centre. We would also like to thank the senior clinical and management team at the Fistula Care Centre for allowing us to work with them on this project.

\section{References}

Abdullah, M., \& Al-Salamah, S. (2009). Impact of comorbidity on outcome among acute non-traumatic surgical patients. Evaluation of Charlson comorbidity index. Saudi Med. J., 30(2), 228-233.

Cant, R., \& Cooper, S. (2010). Simulation based learning in nurse education: systematic review. J. Adv. Nurs., 66(1), 3-15. https://doi.org/10.1111/j.1365-2648.2009.05240.x

Carroll, L. (2004). Clinical skills for nurses in the medical assessment units. Nursing Standard, 18(42), 33-40.

Chen, S., Huang, T., Liao, I., \& Liu, C. (2015). Development and validation of the Simulation Learning Effectiveness. $J$. Adv. Nurs., 71(10). https://doi.org/10.1111/jan.12707

Culebras, J. (2013). Malnutrition in the twenty-first century: An epidemic affecting surgical outcome. Surgical Infections, 14(3), 237-243. https://doi.org/10.1089/sur.2013.9993

Davis, D. (1998). Continuing medical education: global health, global learning. B. M. J., 385, 385-389. https://doi.org/10.1136/bmj.316.7128.385

Hogg, G., \& Miller, D. (2016). The effects of an enhanced simulation programme on medical students' confidence responding to clinical deterioration. B. M. C. Med. Ed., 16(161). https://doi.org/10.1186/s12909-016-0685-2

Huang, G., Gordan, J., \& Schwartzstein, R. (2007). Millenium conference 2005 on medical simulation: a summary report. Simul Healthc, 2(2), 88-95. https://doi.org/10.1097/SIH.0b013e318053e066

Manafa, O., et al. (2009). Retention of health workers in Malawi: Perspectives of health workers and district management. Human Resources for Health, 7(65). https://doi.org/10.1186/1478-4491-7-65

Mooney, J., Chow, C., \& Hillis, G. (2014). Perioperative renal function and surgical outcome. Curr. Opin. Anaesthiol., 27(2), 195-200. https://doi.org/10.1097/ACO.0000000000000054

Munroe, B., et al. (2016). A structured framework improves clinical patient assessment and nontechnical skills of early career emergency nurses: a pre-post study using full immersion simulation. J. Clin. Nurs., 25, 2262-2274. https://doi.org/10.1111/jocn.13284

Muula, A., et al. (2004). Access to continued professional education among health workers in Blantyre, Malawi. African Health Sciences, 4(3), 182-183.

Resuscitation Council, UK (2016). Advanced Life Support. $7^{\text {th }}$ Edition.

Ruesseler, M., et al. (2010). Simulation training improves ability to manage medical emergencies. Emerg. Med. J., 27(10). https://doi.org/10.1136/emj.2009.074518

Theilen, U., et al. (2013). Regular in situ simulation training of paediatric medical emergency team improves hospital reponse to deteriorating patients. Resuscitation, 84(2), 218-222. https://doi.org/10.1016/j.resuscitation.2012.06.027

World Health Organisation (2001). Macroeconomic and health: Investing in health for economic development. Report of the Commission on Macroeconomics and health, Geneva, World Health Organisation. 
World Health Organisation (2014). Country cooperation strategy at a glance Geneva: World Health Organisation, available at: http://www.who.int/countryfocus/cooperation_strategy/ccsbrief_mwi_en.pdf?ua=1

World Health Organisation (2015). United Kingdom: WHO Statistical Profile [pdf] Geneva: World Health Organisation, available at: http://who.int/gho/countries/gbr.pdf

Zendejas, B., Brydges, R., Wang, A., \& Cook, D. (2013). Patient outcomes in simulation based medical education: A systematic review. J. Gen. Intern. Med., 28(8), 1078-1089. https://doi.org/10.1007/s11606-012-2264-5

\section{Copyrights}

Copyright for this article is retained by the author(s), with first publication rights granted to the journal.

This is an open-access article distributed under the terms and conditions of the Creative Commons Attribution license which permits unrestricted use, distribution, and reproduction in any medium, provided the original work is properly cited. 